\title{
Pancreatic pseudocyst-portal vein fistula
}

\author{
Kei Horino, Hiroshi Takamori, Yoshiaki Ikuta, Akira Chikamoto, Takatoshi Ishiko, \\ Toru Beppu, Hideo Baba*
}

Department of Gastroenterological Surgery, Graduate School of Medical Sciences, Kumamoto University, Kumamoto, Japan

Email: "hdobaba@kumamoto-u.ac.jp

Received 29 April 2012; revised 16 June 2012; accepted 28 June 2012

\begin{abstract}
Pancreatic pseudocyst-portal vein fistulae are extremely rare, and cause serious complications, including life-threatening sepsis. We report a 57-year-old man with pancreatic pseudocyst who was admitted to our hospital complaining of prolonged abdominal pain. A pancreatic duct tube was placed into the main pancreatic duct, as the pseudocyst was communicated to the pancreatic duct. He fell into septic shock state two weeks later. An ultrasonography-guided percutaneous drainage of the pseudocyst was performed. The amylase level of drained pus was 80,000 U/l. Pancreatic pseudocyst-portal vein fistula was demonstrated by fisterography. Symptoms disappeared immediately after drainage. No recurrence was observed afterward.
\end{abstract}

Keywords: Pancreatic Pseudocyst; Fistula; Pancreatitis; Portal Vein; Drainage

\section{INTRODUCTION}

Pancreatic pseudocysts are well known as common complications of acute or chronic pancreatitis and complicate the course of chronic pancreatitis in $30 \%$ to $40 \%$ of patients [1-3]. However there have been few reports of pancreatic pseudocyst-portal vein fistulae that cause serious complications, including life-threatening sepsis. We report the case of a patient with a pancreatic pseudocystportal vein fistula required percutaneous drainage as a treatment for the sepsis.

\section{CASE REPORT}

A 57-year-old man was admitted to Kumamoto University hospital complaining of prolonged upper abdominal pain. He had been diagnosted as a chronic panceratitis with a history of heavy alcohol use. Ultrasonography, endoscopic retrograde cholangiopancreatograpy and CT (Figure 1) demonstrated the pancreatic pseudocyst-portal vein fistula with portal thrombosis. As a first treatment, an

${ }^{*}$ Corresponding author. endoscopic retrograde pancreatic duct tube was placed into the main pancreatic duct. We observed the pseudocyst was communicated to the pancreatic duct. He suffered from septic shock with enterococcus in blood culture two weeks later. Because septic state was not improved by the conservative treatment with antibiotics and CT revealed that the pancreatic pseudocyst was increasing, ultrasonography-guided percutaneous drainage into the pseudocyst was performed. An amount of $40 \mathrm{ml}$ of pus was drained. The amylase level of this fluid was 80,000 U/l. Fisterography demonstrated the pancreatic pseudocyst connected to the portal vein (Figure 2). Both high fever and upper abdominal pain disappeared immediately. A CT performed three weeks later demonstrated a significant reduction of the pseudocyst. Neither recurrence of abdominal pain nor fever was observed afterward.

\section{DISCUSSION}

Only 17 cases of pancreatic pseudocyst connecting with the portal venous system have been reported [4-6]. Several reports indicated that activated pancreatic enzymes in the pseudocyst might cause inflammation and directly

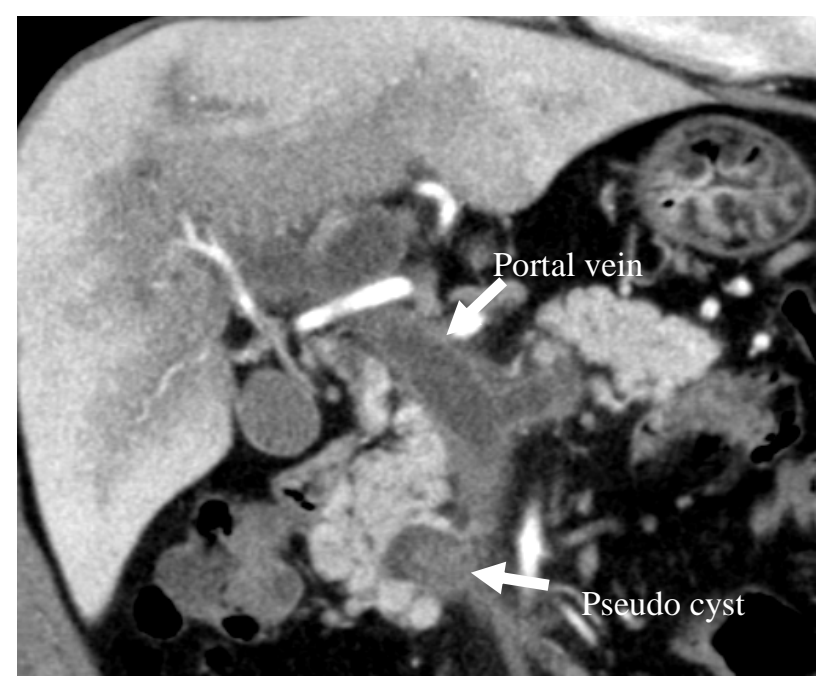

Figure 1. Abdominal computed tomography: the pancreatic pseudocyst-portal vein fistula (white arrow) with thrombosis. 


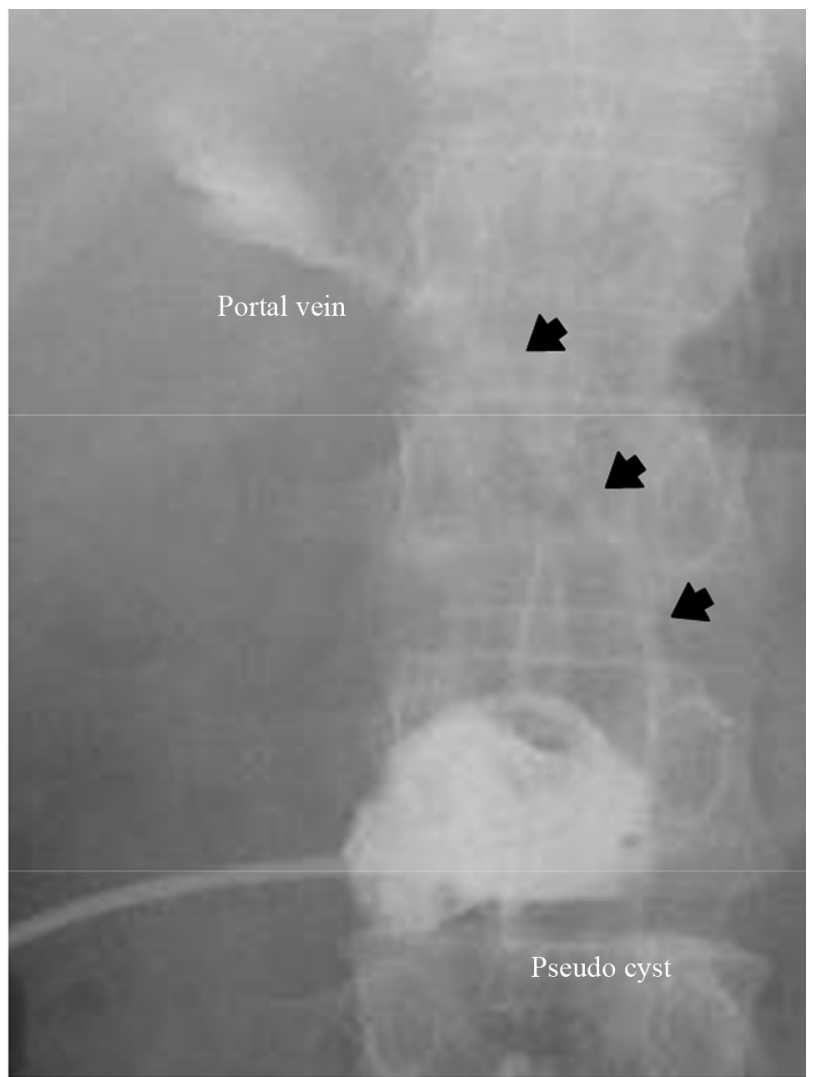

Figure 2. Fisterography: the pancreatic pseudocyst connected to portal vein (black arrow).

contribute to cyst wall fragility and failure. These enzymes also cause erosion of the portal wall and induce venous thrombosis $[4,7]$. Spontaneous rupture of the pancreatic cyst occurred in about 5\% of patients [7]. Most patients suffering from pancreatic pseudocyst-portal vein fistulae are asymptomatic or complained of mild abdominal pain. However, some patients have severe, life-threatening complications, such as massive bleeding in the digestive tract, septic shock, disseminated fat necrosis and intramedullary bone necrosis [4].

In this case report, we report a case of pancreatic pseudocyst-portal vein fistula in which the patient fell into septic shock state, which was improved by Ultrasonography-guided percutaneous drainage.

\section{REFERENCES}

[1] Habashi, S. and Draganov, P.V. (2009) Pancreatic pseudocyst. World Journal of Gastroenterology, 15, 38-47. doi:10.3748/wjg.15.38

[2] Khanna, A.K., Tiwary, S.K. and Kumar, P. (2012) Pancreatic pseudocyst: Therapeutic dilemma. International Journal of Inflammation, 2012, Article ID: 279476. doi:10.1155/2012/279476

[3] Lankisch, P.G., Weber-Dany, B., Maisonneuve, P. and Lowenfels, A.B. (2012) Pancreatic pseudocysts: Prognostic factors for their development and their spontaneous resolution in the setting of acute pancreatitis. Pancreatology, 12, 85-90.

[4] Yoon, S.E., Lee, Y.H., Yoon, K.H., Choi, C.S., Kim, H.C. and Chae, K.M. (2008) Spontaneous pancreatic pseudocyst-portal vein fistula presenting with pancreatic ascites: Strength of mr cholangiopancreatography. British Journal of Radiology, 81, e13-e16. doi:10.1259/bjr/77979574

[5] Hammar, A.M., Sand, J., Lumio, J., Hirn, M., Honkonen, S., Tuominen, L. and Nordback, I. (2002) Pancreatic pseudocystportal vein fistula manifests as residivating oligoarthritis, subcutaneous, bursal and osseal necrosis: A case report and review of literature. Hepato-Gastroenterology, 49, 273-278.

[6] Noh, R. and Kim, H.J. (2010) A pancreatic pseudocyst-portal vein fistula closed by endoscopic pancreatic stent insertion. Gastrointestinal Endoscopy, 72, 1103-1105. doi:10.1016/j.gie.2010.02.019

[7] Takayama, T., Kato, K., Sano, H., Katada, N. and Takeichi, M. (1986) Spontaneous rupture of a pancreatic pseudocyst into the portal venous system. American Journal of Roentgenology, 147, 935-936. 